\title{
Phase transition in quantum tunneling and exact statistical mechanics for a model of parametrized double-well potential
}

\author{
Fernand Naha Nzoupe ${ }^{1}$, Alain Dikande ${ }^{2}$, and Clement Tchawoua ${ }^{1}$ \\ ${ }^{1}$ University of Yaounde 1 Faculty of Sciences \\ ${ }^{2}$ University of Buea
}

June 20, 2020

\begin{abstract}
A model for one-dimensional bistable systems characterized by a deformable double-well energy landscape, is introduced in order to investigate the effect of shape deformability on the order of phase transition in quantum tunneling, and on the quasiexact integrability of the classical statistical mechanics of these systems. The deformable double-well energy landscape is modelled by a parametrized double-well potential possessesing two fixed degenerate minima and a constant barrier height, but a tunable shape of its walls which affects the confinement of the two wells. It is found that unlike bistable models involving the standard $\$ \backslash$ phi $^{\wedge} 4 \$$-field model for which the transition in quantum tunneling is predicted to be strictly of second order, a parametrization of the double-well potential also favors a first-order transition occurring above a universal critical value of the shape deformability parameter. The partition function of the model is constructed within the framework of the transfer-integral formalism, with emphasis on low-lying eigenstates of the transfer-integral operator. A criteria for quasi-exact integrability of the partition function is formulated, in terms of the condition for possible existence of exact eigenstates of the transferintegral operator. The quasi-exact solvability condition is obtained analytically and from this, some exact eigenstates are derived at several temperatures. The exact probability densities obtained from the analytical expressions of the groundstate wavefunctions at different temperatures, are found to be in excellent agreement with the probability density obtained from numerical simulations of the Langevin equation.
\end{abstract}

\section{Hosted file}

artnaha.pdf available at https://authorea.com/users/335318/articles/461149-phase-transitionin-quantum-tunneling-and-exact-statistical-mechanics-for-a-model-of-parametrized-doublewell-potential 
figures/nahafig1/nahafig1-eps-converted-to.pdf 
figures/nahafig2/nahafig2-eps-converted-to.pdf 
figures/nahafig3/nahafig3-eps-converted-to.pdf 
figures/nahafig4/nahafig4-eps-converted-to.pdf 
figures/nahafig5/nahafig5-eps-converted-to.pdf 
figures/nahafig6/nahafig6-eps-converted-to.pdf 
figures/nahafig7/nahafig7-eps-converted-to.pdf 
figures/nahafig8/nahafig8-eps-converted-to.pdf 
figures/nahafig9/nahafig9-eps-converted-to.pdf 
figures/nahafig10/nahafig10-eps-converted-to.pdf 
figures/nahafig11/nahafig11-eps-converted-to.pdf 
figures/nahafig12a/nahafig12a-eps-converted-to.pdf 